\title{
Neutron Effects on Short Circuit Currents of Op Amps and Consequences
}

\author{
F. J. Franco, Y. Zong, Juan Casas-Cubillos, M. A. Rodríguez-Ruiz, and J. A. Agapito
}

\begin{abstract}
Discrete bipolar operational amplifiers were irradiated with neutrons in order to study the evolution of the short circuit currents. Also, this paper explores the effect of the reduction of this current in devices based on operational amplifiers.
\end{abstract}

\section{Index Terms}

Bipolar operational amplifiers, COTS, displacement damage, radiation tolerance.

\section{INTRODUCTION}

$\mathbf{T}$ HIS harsh environment expected in the cryogenic system of the LHC of CERN will affect the devices present in the electronic instrumentation. Unlike other radiation environments, as space or nuclear plants, displacement damage will be very important, so neutron tests on devices built in bipolar technologies must be carried out in order to select the most tolerant commercial devices. Therefore, a deep study of the evolution of all the parameters of these devices under neutron radiation has been done. These experiences have provided many details about the behavior of parameters as input offset voltage, input bias currents, open loop gain, frequency parameters, etc. However, the purpose of this paper is to discuss the effect of the displacement damage on the short circuit currents since we believe that it is an interesting parameter whose behavior is not well known. Furthermore, the consequences of the degradation of this parameter will be determined in feedback networks and other devices where operational amplifiers are integrated.

Operational amplifiers built in bipolar technologies usually have three main stages: Input, gain and output. The first stage is a differential pair, whether bipolar or JFET, and the gain stage can be another bipolar differential pair or a Darlington transistor loaded with a current source [1].

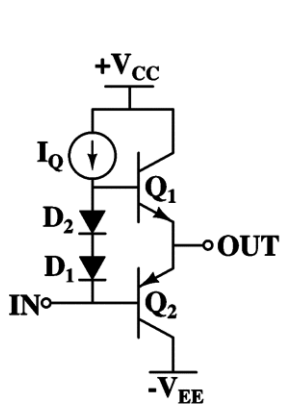

(a)

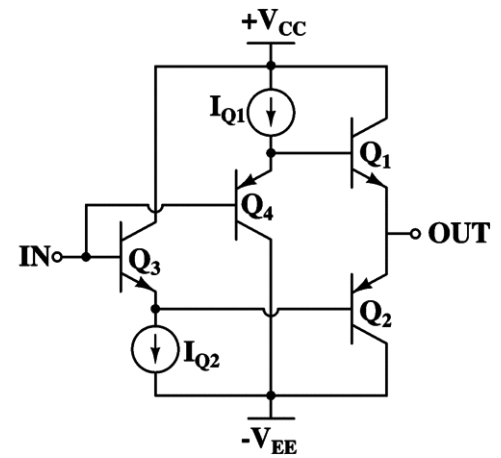

(b)

Figure 1. Different kinds of class AB output stages. (a) "Push-pull" network and (b) improved Class AB output stage with complementary emitter follower crossover distortion compensation.

The output stage of low signal bipolar op amps (power op amps are not included in this work) should have the following characteristics: First of all, it must be very linear to minimize the distortion and, second, the consumption should not be very high. Therefore, class AB complementary output stages are widely used [2], [3]. Fig. 1a shows the "push-pull" network, the most simple network belonging to this kind of output stages. This output stage is complementary since there are NPN and PNP transistors that work in turns depending on the sign of $\mathrm{V}_{I N}$. The couple of diodes is used as a DC level shifter to linearize the input-output function. In fact, if the op amp is loaded and the input voltage is negative, the active transistor is $\mathrm{Q}_{2}$ so $V_{O U T}=V_{I N}+V_{B E, Q 2} \approx V_{I N}+0.7\left(V_{I N}<0\right)$. If the input voltage is positive, $\mathrm{Q}_{1}$ is working and $V_{O U T}=$ $V_{I N}+V_{D 1}+V_{D 2}-V_{B E, Q 1} \approx V_{I N}+0.7$. Since both expressions are equal, the input-output function is continuous and linear.

This work was supported in part by the cooperation agreement K476/LHC between CERN and UCM, the Spanish research agency CICYT (FPA2002-00912), and ITN.

F. J. Franco and J. A. Agapito are with the Departamento de Fisica Aplicada III, Facultad de Ciencias Físicas, Universidad Complutense de Madrid, 28040 Madrid, Spain (e-mail: monti@fis.ucm.es).

J. Casas-Cubillos and M. A. Rodríguez-Ruiz are with the CERN, AT Division, Geneva, Switzerland (e-mail: juan.casas-cubillos@cern.ch).

Y. Zong is with the Departamento de Fisica Aplicada III, Facultad de Ciencias Físicas, Universidad Complutense de Madrid, 28040 Madrid Spain, on leave of absence from Wuhan Institute of Chemical Technology, China (e-mail: yzong@ fis.ucm.es). 


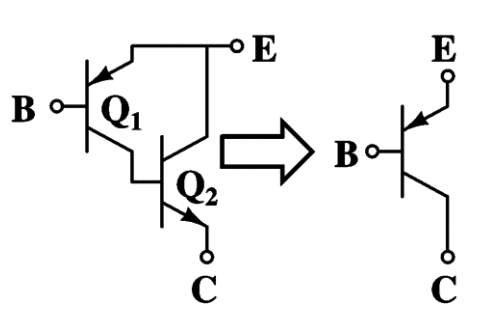

(a)

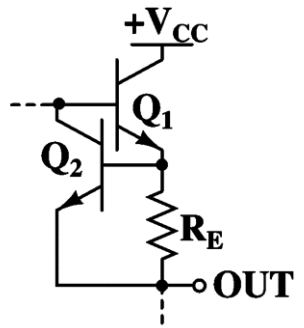

(b)

Figure 2. (a) False PNP transistor to increase the current gain of PNP transistors. Also, (b) shows the use of a feedback network to limit the output current. If $\mathrm{Q}_{1}$ emitter current is too high, $\mathrm{Q}_{2}$ base-emitter voltage increases, taking current from $\mathrm{Q}_{1}$ base.

However, the pure "push-pull" network is found in very few types of operational amplifiers, like LF351. Other amplifiers, as OP-27, have a modified output stage (Fig. 1b), with distortion compensation [3]. In this stage, $V_{I N} \approx V_{O U T}$. Moreover, several improvements are introduced in many output stages [2]: Use of a Darlington pair or false PNPs (Fig. 2a) to improve the low current gain of PNP transistors; replacement of $\mathrm{D}_{1} \& \mathrm{D}_{2}$ by other DC level shifter based on the BE junctions of bipolar transistors or resistor networks; resistors between $\mathrm{Q}_{1} \& \mathrm{Q}_{2}$ emitters to limit the current (Fig. 2b) (e.g., the popular $\mu \mathrm{A} 741$, etc. Also, the output stage of some amplifiers shares some of their elements with other stages. For example, in LF351, $\mathrm{I}_{Q}$ in Fig. 1a is the current source that also biases the gain stage. In any case, the functioning is similar to the "push-pull" configuration.

\section{RADIATION DATA ON DISCRETE OPERATIONAL AMPLIFIERS}

The short circuit currents of an operational amplifier is defined as the highest current that the device can provide or absorb. Thus, there are two values, positive and negative, depending on the fact that the op amp is working as a current source or a sink.

\section{A. Tested Devices, Radiation Environments, and Test Set-Up}

A set of low signal operational amplifiers were irradiated in a special facility at the Portuguese Research reactor [4]. This facility has been designed to reach the total radiation dose calculated in the LHC cryogenic system for 10 years of activity. In five sessions of $12 \mathrm{~h}$ each, the neutron fluence in the center or the cavity rose up to $5 \cdot 10^{13} \mathrm{n} / \mathrm{cm}^{2}$ and the background gamma radiation dose was between $1-3 \mathrm{kGy}(\mathrm{Si})$.

The energy spectrum of the neutron beam was similar to that of the ${ }^{235} \mathrm{U}$ fission after removing the component of thermal neutrons. According to the calculations performed in the Portuguese Research Reactor [5], the correction factor value is 1.28. In other words, all the neutron fluence values shown in this paper can be expressed in the standard unit of $1 \mathrm{MeV}$-neutrons $/ \mathrm{cm}^{2}$ just multiplying it by 1.28 .

Tested amplifiers were OP-07, OP-27 \& OP-77, from Analog Devices, LF351, from Linear Technology, and OPA227, OPA277, OPA111, OPA132, OPA602, OPA606, OPA627, and TLE2071, from Burr-Brown and Texas Instruments. Their characteristics can be found on manufacturers' web pages. All these amplifiers are built in bipolar technologies with a variety of features: Bipolar, JFET or DiFET input; lower or higher open loop gain; broad frequency response, etc. In some cases, the internal topology is public and the output stages are similar to the models shown in Section I. During the irradiation, their leads were shorted, as standard protocols suggest [6]. The devices received a neutron fluence between $1.75 \cdot 10^{13} \mathrm{n} / \mathrm{cm}^{2} \& 10^{14}$ $\mathrm{n} / \mathrm{cm}^{2}$, being the vestigial gamma dose between $1450 \& 2700 \mathrm{~Gy}(\mathrm{Si})$. Neutron fluence values were measured with ${ }^{58} \mathrm{Ni}$ foils and total ionizing dose with an ionization chamber.

The radiation tests were carried out in two rounds: First, a preliminary test of three or four samples of every amplifier was done and, later, a second test on about twenty samples of the most tolerant devices. This is the reason that there are more data about OPA627 and TLE2071 than about any other device since their results after the preliminary test were very promising.

After checking that the devices were still functional after the irradiation, by measuring different parameters as input offset voltage, open loop gain, etc., the values of the short circuit currents were measured following this standard procedure: First, the amplifier is biased by a pair of power supplies with values of $\pm 10 \& \pm 15 \mathrm{~V}$. The inverting input is grounded and the noninverting one is connected to a voltage source whose value can switch between $\pm 5 \mathrm{~V}$. Thus, the amplifier is easily led to positive and negative saturation. The output of the amplifier is connected to an accurate multimeter (Keithley 2002), ready to store in a buffer the values of the current, and, finally, to a switch joining the output of the amplifier to ground. The purpose of this switch is to connect the amplifier output to ground only for very few seconds and prevent heating effects. Afterwards, the highest current value is chosen among the data array saved in the multimeter buffer. This procedure is repeated changing the input voltage and the value of the power supplies.

These parameters were measured before and after the irradiation. Due to safety reasons, the short circuit current of irradiated amplifiers was determined only after the deactivation of radioactive isotopes. This fact took about one month later and, during this period of time, the devices were isolated at room temperature. 


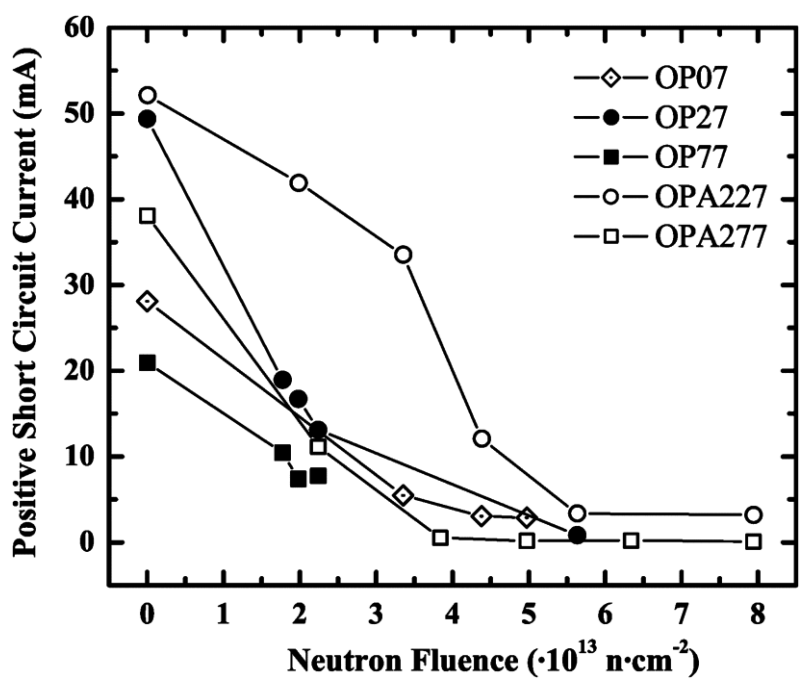

Figure 3. Positive short circuit current of several discrete commercial operational amplifiers.

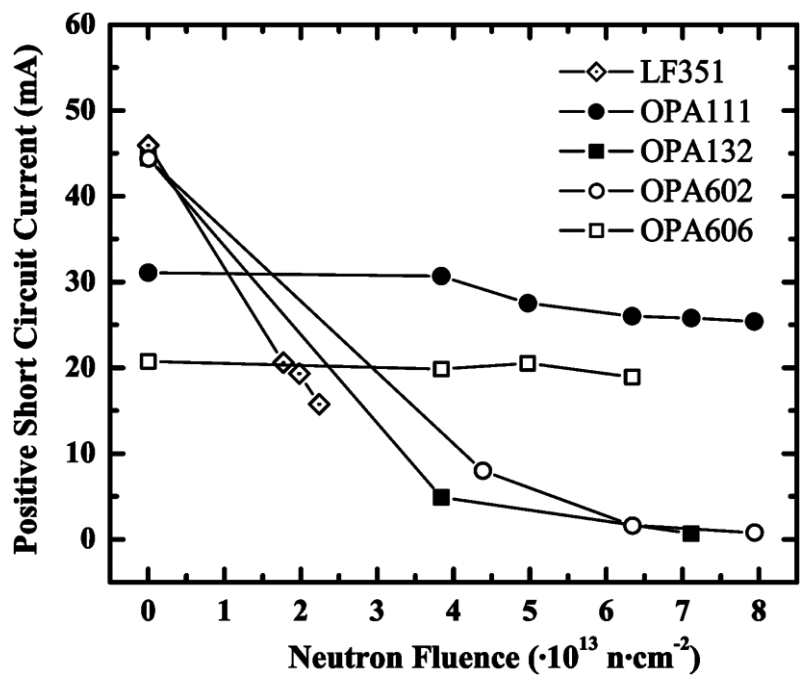

Figure 4. Positive short circuit current of several discrete commercial operational amplifiers.

\section{B. Effects of Radiation on Short Circuit Currents}

After the safety period, the positive and negative short circuit currents of the operational amplifiers were measured and compared with the initial one. Figs. 3-5 are related to the positive short circuit current in some of the tested devices with power supplies of $\pm 15 \mathrm{~V}$. These figures show a decrease of the current values as the neutron fluence grows. The exceptions to this behavior are OPA111 and OPA606 amplifiers. Finally, Figs. 6-8 show the evolution of the negative short circuit current. Like positive currents, the values of these parameters drop in proportion to the neutron fluence.

Since most tested devices could work after the irradiation, there are values of positive and negative short circuit currents in almost all the devices. The only exceptions were an OP-27 sample that received a neutron fluence of $5.6 \cdot 10^{13} \mathrm{n} / \mathrm{cm}^{2}$ and an OPA606 op amp, which could not tolerate a neutron fluence of $6.4 \cdot 10^{13} \mathrm{n} / \mathrm{cm}^{2}$. In any case, the main sign of their destruction was that their outputs were saturated whatever the input was. Therefore, at least one of the couple of values was successfully determined. This is why, in some devices, the set of positive values is larger than the negative one, or vice versa.

\section{Influence of Power Supplies in the Short Circuit Current}

In an ideal operational amplifier, the short circuit currents hardly depend on the value of the power supplies. However, this property is no longer present in a great deal of irradiated amplifiers. Fig. 9 shows the ratio between the positive short circuit currents at $\pm 15 \mathrm{~V} \& \pm 10 \mathrm{~V}$. In the not irradiated samples, this ratio is close to 1 but it soars in proportion to the neutron fluence. This phenomenon is more important in the OP-27 and OPA227, which have the same internal topology despite they are manufactured by different companies. 


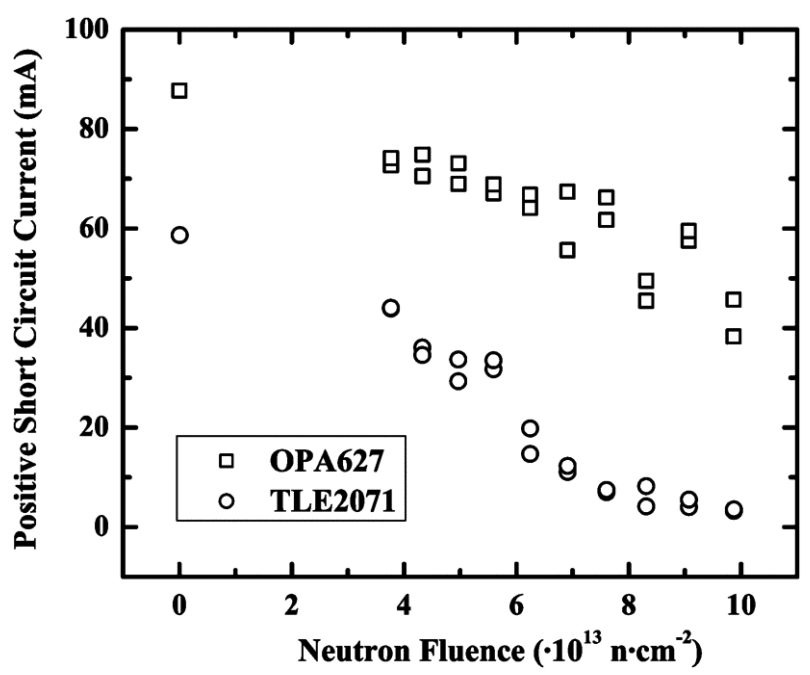

Figure 5. Positive short circuit current of the most rad-tol operational amplifiers, OPA627 and TLE2071.

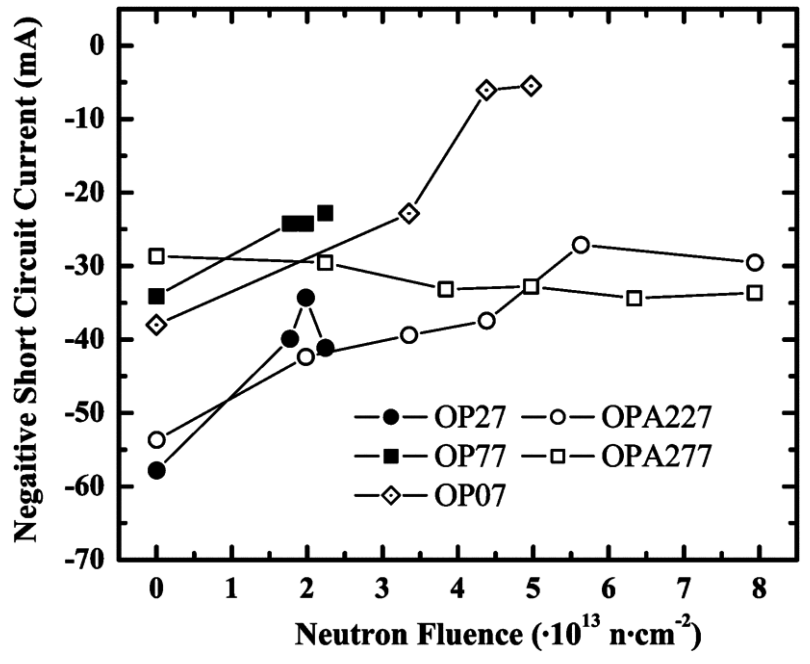

Figure 6. Negative short circuit current of several discrete commercial operational amplifiers.

This increase was not found in the negative short circuit current, where the values kept independent on the power supplies. The only exception was OP-07, whose value of $I_{S h C C, 15 V} / I_{S h C C, 10 V}$ rises up to 5 at $5 \cdot 10^{13} \mathrm{n} / \mathrm{cm}^{2}$.

\section{DISCUSSION}

The decrease of the short circuit currents has been found in all the amplifiers except in few devices. Therefore, the reason must be related to the topology and not to the manufacturing technology. The following sections develop this idea to explain the experimental results shown in the previous section.

\section{A. Causes of Short Circuit Current Diminution}

The theoretical value of the short circuit current is calculated with the following three postulates:

1) gain stage can be replaced by a Thevenin network

2) the output value of the gain stage switches between $+\mathrm{V}_{C C}$ and $-\mathrm{V}_{E E}$

3) the output stage is connected to ground by a load, $\mathrm{R}_{L}$, very small but never a short circuit

These postulates help to simplify the mathematical calculi. Let us study the typical "push-pull" configuration with the previous assumptions (Fig. 10). It is loaded with a resistor $\mathrm{R}_{L}$ and the gain stage has been replaced by its equivalent network. Now, we will suppose that $R_{L} \rightarrow 0$ so the amplifier must provide or absorb the highest output current. Since this value is not infinite, it is obvious that $V_{O U T} \rightarrow 0$.

Now, let us accept that $\mathrm{V}_{I N}$ is the highest possible value, which would be close to $+\mathrm{V}_{C C}$. Due to $V_{\text {OUT }} \sim 0 \mathrm{~V}, \mathrm{Q}_{2}, \mathrm{D}_{1} \&$ $\mathrm{D}_{2}$ are cut off. Therefore, the current that flows to the base of $\mathrm{Q}_{1}$ is $\mathrm{I}_{Q}$ so the short circuit current is

$$
I_{S h C C, P o s}=I_{E, Q 1}=\left(h_{F E, 1}+1\right) \cdot I_{Q}
$$




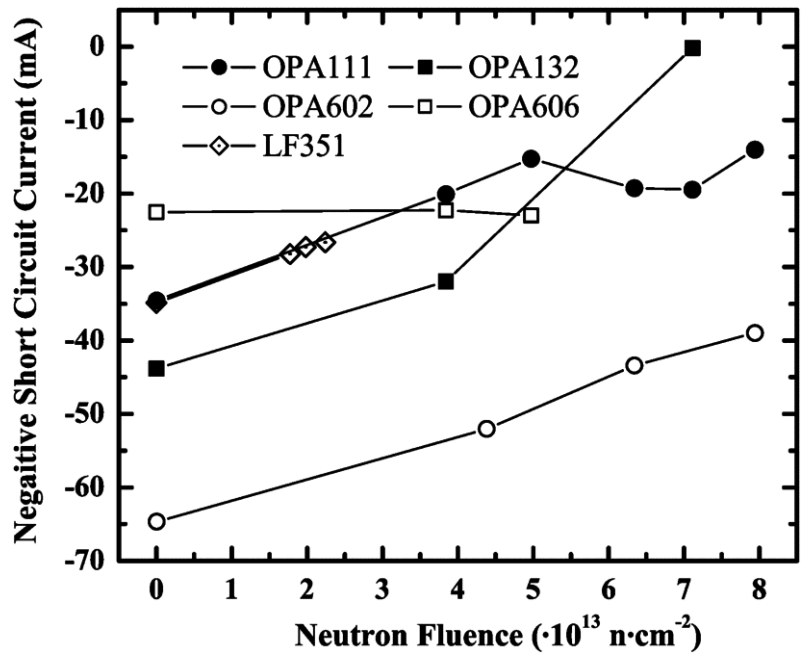

Figure 7. Negative short circuit current of several discrete commercial operational amplifiers.

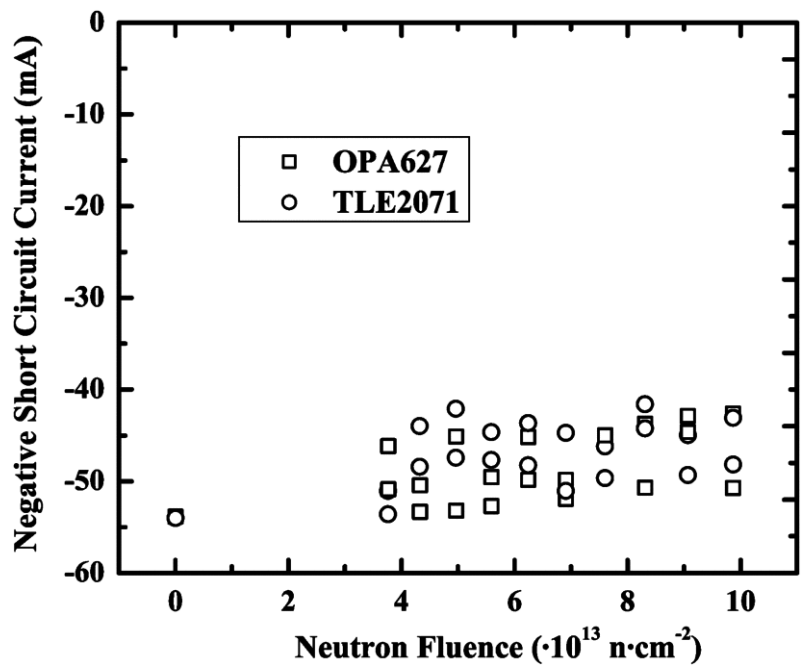

Figure 8. Negative short circuit current of the most rad-tol operational amplifiers, OPA627 and TLE2071.

This is $\mathrm{Q}_{1}$ emitter current. Unfortunately, this statement has a weak point. $\mathrm{I}_{Q}$ is supposed to belong only to the output stage but this is not always true, e.g., in LF351, $\mathrm{I}_{Q}$ is the current source that biases the gain stage. Therefore, the whole current cannot go to the output stage since the amplifier would stop working. However, Eq. 1 must be interpreted as a top value of $I_{S h C C, P O S}$ rather than the exact one. Other amplifiers, such as OPA627, have a current source exclusively devoted to bias the output stage so Eq. 1 is a good estimate of the actual value of positive short circuit current.

In the case of a negative input voltage, the statement is more complex. $\mathrm{Q}_{1} \& \mathrm{Q}_{2}$ are active so we have to do one additional simplification: The fraction of current going along $\mathrm{Q}_{1}$ base is $\varepsilon \cdot I_{Q}, 0<\varepsilon<1$. After some steps, the following result appears:

$$
\begin{aligned}
I_{S h C C, N e g}= & \left(h_{F E, 2}+1\right) \cdot\left(\frac{V_{E E}}{R_{G}}-(1-\varepsilon) \cdot I_{Q}\right) \\
& -\varepsilon\left(h_{F E, 1}+1\right) \cdot I_{Q}
\end{aligned}
$$

where $-\mathrm{V}_{E E}$ is the negative power supply. If this method were used to calculate the short circuit current of other stages, similar results would be found, e.g., the output stage of Fig. 1 b has the current values shown in Eq. $3 \& 4$

$$
\begin{aligned}
& I_{S h C C, \text { Pos }}=\left(h_{F E, Q 1}+1\right) \cdot I_{Q 1} \\
& I_{S h C C, \text { Neg }}=\left(h_{F E, Q 2}+1\right) \cdot I_{Q 2}
\end{aligned}
$$

If Darlington pairs or false PNP were present in the network instead of simple transistors, $\mathrm{h}_{F E, Q 1}$ or $\mathrm{h}_{F E, Q 2}$ values would be replaced by the actual gain of the pair. In any case, we can realize that the short circuit currents depend on the current gains 


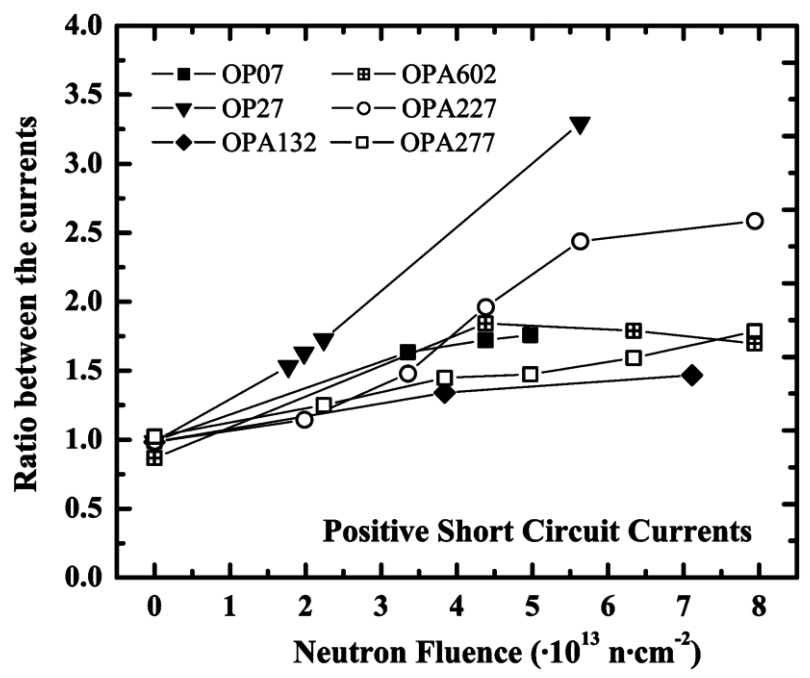

Figure 9. Ratio between the values of the positive short circuit currents with power supplies values of $\pm 15 \mathrm{~V} \& \pm 10 \mathrm{~V}$.

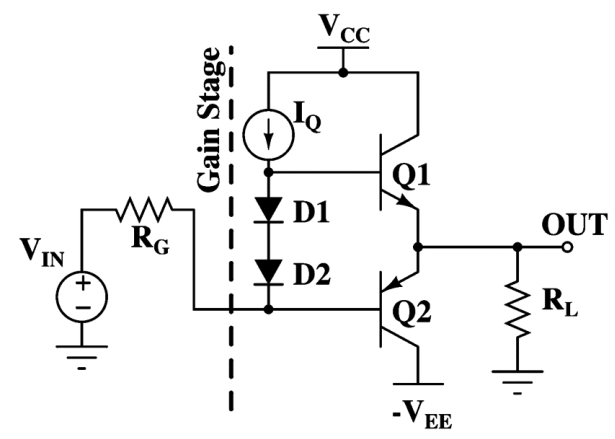

Figure 10. Output stage loaded with a resistor $R_{L}$. Gain stage has been replaced by the equivalent Thevenin network.

of the transistors of the output stage. This important result is also valid in the protection network shown in Fig. $2 \mathrm{~b}$, as some SPICE simulations showed.

Thus, the reduction of short circuit current is explained. Displacement damage will reduce the values of $\mathrm{h}_{F E, Q 1} \& \mathrm{~h}_{F E, Q 2}$ due to the drop of the lifetime of minority carriers [7] so both output currents must decrease. Additional effects, like the growth of collector and base resistances, can be added to the decrease of minority carrier's lifetime to worsen $\mathrm{h}_{F E, X}$.

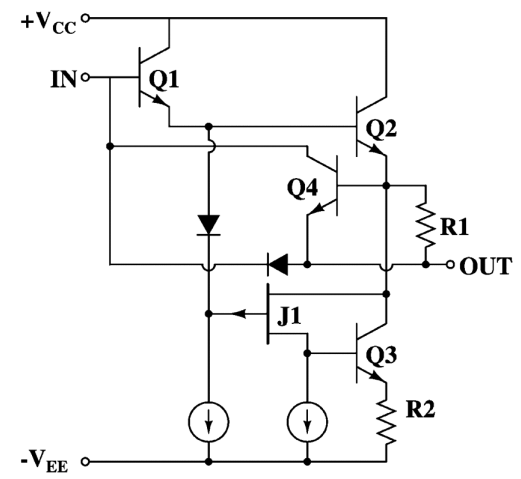

Figure 11. Output stage of OPA606, according to its manufacturer.

An interesting fact to deal with is the high tolerance of OPA111 and OPA606. Unfortunately, the internal topology of the first amplifier is unknown but, on the contrary, OPA606 topology is public. As we might guess, its output stage amplifiers (Fig. 11) have some differences from those present in the other. In this network, there are pairs of transistors with very high gain (Darlington and a variation of false PNP). The short circuit current limitation is done by means of two resistors $\mathrm{R}_{1} \&$ $\mathrm{R}_{2}$ between the output stage and the output of the amplifier. Even though the pairs of transistors are degraded by displacement damage, the transistor pairs could be able to provide a high output current due to its high gain configuration. Indeed, unless 
the transistors are extremely degraded, this current is limited only by the resistors, insensitive to radiation. Thus, the tolerance of OPA606 is explained.

\section{B. Dependence on the Power Supply Value}

The second point of interest is the increasing dependence of the short circuit currents on the power supply voltages. Other consequence of the displacement damage in bipolar transistors is the decrease of the Early voltage [8]. Actually, Eq. 1-4 should be multiplied by a factor $\left(1+V_{C C} / V_{A X}\right)$, being $V_{C C}$ (or, in other cases, $\left.V_{E E}\right)$ the supply voltage and $V_{A X}$ the Early voltage of $Q_{X}$. Thus, the relationship between $+V_{C C} \& I_{S h C C}$ appears immediately.

Nevertheless, there is an indirect mechanism related to the decrease of Early voltage that has even more importance. The nature of $\mathrm{I}_{Q}$ of Fig. 1 is usually unknown but, in most cases, it is a bipolar mirror current [2]. The output transistor of this source shows Early effect too so the influence of $\mathrm{I}_{Q}$ on the supplies is transmitted into the output current after being multiplied by the gain of the transistor $\mathrm{Q}_{1}$.

\section{Displacement Damage or TID?}

The tested samples received a large neutron fluence and a vestigial gamma radiation of $1.5-3 \mathrm{kGy}$. It is also known that the transistor gain decreases with the ionizing radiation so the reduction of the short circuit current might have been attributed also to this kind of radiation.

However, there are some points that clears up this question. A previous ${ }^{60}$ Co test performed at the Portuguese Research Reactor on samples of some of the irradiated devices (LF351, OPA111, OP27, OP77, and TLE2071) showed that they were functional after receiving $18 \mathrm{kGy}(\mathrm{Si})$ for $60 \mathrm{~h}$ and that the short circuit currents had not been as affected as during the neutron irradiation. Let us keep in mind that $18 \mathrm{kGy}$ is a TID value six or ten times higher than those measured in the neutron facility. The most important degradation was observed in LF351, where a decrease of 46 to $30 \mathrm{~mA}$ was found in $I_{S h C C}$, value that must be compared to $16 \mathrm{~mA}$, value measured in a sample that received $2.2 \cdot 10^{13} \mathrm{n} / \mathrm{cm}^{2}$ and only $1.7 \mathrm{kGy}$. In the rest of the amplifiers, the decrease is only of $5 \%-10 \%$ of the original value. Besides, unlike the results of the neutron test, no influence of power supplies was appreciated.

The influence of the displacement damage can be also estimated by some calculations from the Messenger-Ash's law [7], [8], $h_{F E}^{-1}=h_{F E, 0}^{-1}+\Phi / K_{\Phi}$. Let us focus on the case of the positive short circuit current. First, we shall assume that the base doping of the NPN transistor of Fig. 1 is about $10^{16} \mathrm{~cm}^{-3}$, typical value found in bipolar transistors, leading to a resistivity value about $1 \Omega \cdot m$ [9]. Tested op amps do not belong to the class of RF devices so we guess that they are built with general purpose bipolar transistors, whose value of $\mathrm{f}_{T}$ is found between 50 and $500 \mathrm{MHz}$ [10]. Finally, devices are supposed to be unbiased during the irradiation, which is the case of largest damage. Accepting these three postulations, values of $\mathrm{K}_{\Phi}$ between $7.1 \cdot 10^{13} \& 7.1 \cdot 10^{14}$ neutron $(1 \mathrm{MeV}) / \mathrm{cm}^{2}$ would be eventually obtained. These values are, as expected, in the order of the neutron fluence levels reached during the tests so the behavior of the short circuit currents could be explained.

Nevertheless, despite we have demonstrated that the main agent of degradation in our experiments was the displacement damage, playing the ionizing radiation a secondary role, the effect of TID could be important in the case of other popular operational amplifiers. An example of this is the degradation of LF351, shown in this paper, although not as important as that caused by the neutron radiation, by no means negligible, e.g., some authors have pointed out to a significant diminution of the value of the short circuit current in some devices after TID tests, like those shown by Bonora about LM124 [11]. Finally, it is obvious that the evolution of the short circuit currents could be also influenced by the effect of the enhancement at low dose rate.

\section{FURTHER CONSEQUENCES OF THE DECREASE OF SHORT CIRCUIT CURRENTS}

\section{A. Irradiated Amplifiers in a Resistor Feedback Network}

The short circuit current is a parameter that is not usually measured in irradiated op amps but its reduction may affect the correct functioning of electronic systems, e.g., let us suppose that an op amp is placed in a noninverting configuration with two resistors of 10 and $1 \mathrm{k} \Omega$ and the output voltage sweeps between $\pm 10 \mathrm{~V}$. Therefore, the op amp must provide or absorb $\pm 1 \mathrm{~mA}$. In Fig. 4, we showed an OPA602 op amp that received $7.9 \cdot 10^{13} \mathrm{n} / \mathrm{cm}^{2} \& 2700 \mathrm{~Gy}$. At this radiation level, the values of most $\mathrm{DC}$ and $\mathrm{AC}$ parameters are very interesting: $V_{O S}=-6.8 \mathrm{mV}, I_{B+}=30 \mathrm{pA}$, slew rate $=6.3 \mathrm{~V} \mu \mathrm{s}$, openloopgain $=85 \mathrm{~dB}$, etc. These values are very good so the amplifier would be thought as rad-tolerant. However, its output hardly provides 0.75 $\mathrm{mA}$. Therefore, the circuit where it is placed will not work correctly since the amplifier output would saturate at $8.25 \mathrm{~V}$, unable to reach the largest positive values and making the whole electronic system useless.

\section{B. Operational Amplifiers in Complex Devices}

Operational amplifiers are widely used to build complex devices. During neutron irradiations, some devices showed a degradation that can be attributed to the diminution of short circuit current. These devices are digital-to-analog converters, instrumentation amplifiers and precision voltage references. They were irradiated in the same facility as the discrete operational amplifiers. Some parameters were measured during the irradiation but other were characterized before the test and after the safety period. 
1) Digital-to-Analog Converters (DAC703): This device is manufactured by Texas Instruments. It is a 16-bit parallel input $\mathrm{D} / \mathrm{A}$ converter, with voltage output and built in bipolar technology. It is based in the R/2R ladder network and needs an operational amplifier working as a current-to-voltage converter, loaded with a $5 \mathrm{k} \Omega$ resistor. During the neutron tests, a PC system measured the DAC input-output function every ten minutes, ending the test at a total radiation dose of $3.25 \cdot 10^{13} \mathrm{n} / \mathrm{cm}^{2}$ \& $1.27 \mathrm{kGy}(\mathrm{Si})$.

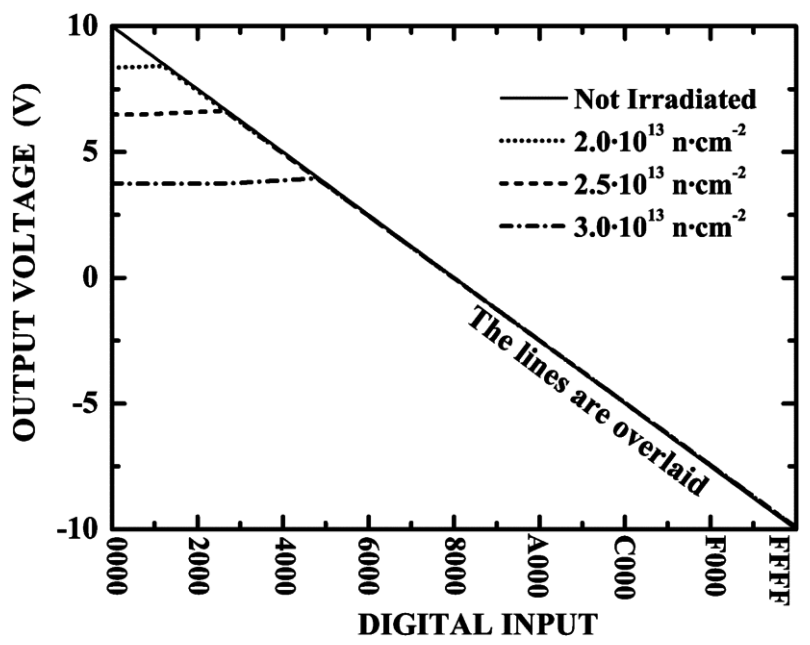

Figure 12. Input-output function of DAC703KH at several values of neutron fluence. Input value is written in hexadecimal format.

At the beginning, that function was a straight line starting at $10 \mathrm{~V}$ with $0000_{H}$ and finishing at $-10 \mathrm{~V}$ with $1111_{H}$. However, as the irradiation takes place, the relation became as Fig. 12 shows. The initial straight line has been split into two parts: A horizontal line for the lowest input values, and the theoretical output for the largest ones. The reason is also related to the diminution of the positive short circuit current of the output opamp. If the output voltage is $10 \mathrm{~V}$, the internal op amp must provide $2 \mathrm{~mA}$ to bias the feedback resistor but, if the irradiated op amp could not, the output would saturate at a voltage lower than $10 \mathrm{~V}$. This fact was confirmed after the irradiation since a value of $0.97 \mathrm{~mA}$ of highest positive output current was measured, more than twenty times smaller than the initial value $(21 \mathrm{~mA})$. On the contrary, the negative short circuit current had hardly changed $(\sim 25 \mathrm{~mA})$ so this explains that the negative output values were not affected. Finally, it is very interesting to point out to the fact that the positive short circuit current depends on $+\mathrm{V}_{C C}$ in the irradiated devices. Thus, if this supply increases from 15 up to $18 \mathrm{~V}$, the short circuit current does as well, shifting from 0.97 to $1.60 \mathrm{~mA}$.

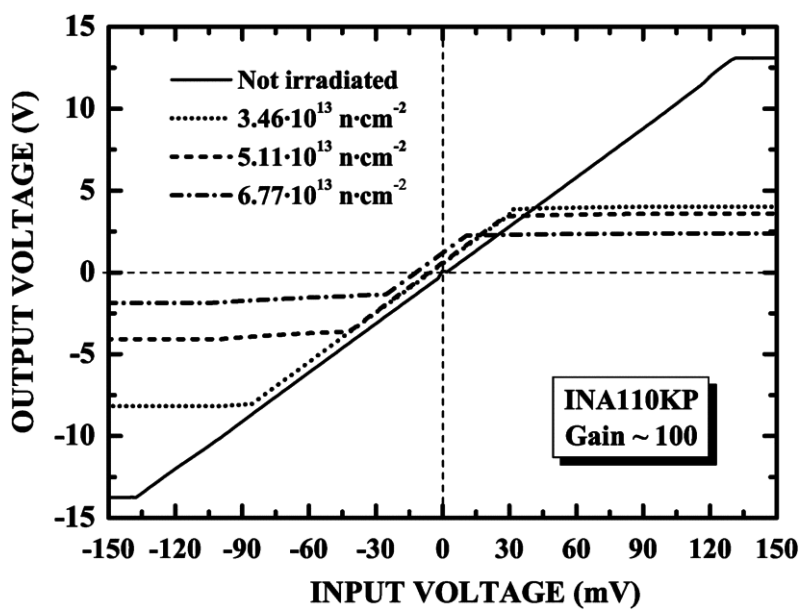

Figure 13. Input and output function of several samples of irradiated INA110KP.

2) Instrumentation Amplifiers (INA110, INA111): These devices are built using the classical three opamp network [10], [12]. Some irradiated samples of both devices show a large decrease of the saturation voltages after being irradiated (Fig. 13). This decrease cannot be attributed to the shift of saturation voltage swing, since it hardly changes in the irradiated operational amplifiers of Section II.

On the contrary, the diminution of short circuit current plays a large role since the internal op amps are loaded with 10-20 $\mathrm{k} \Omega$ resistors. Therefore, the appearance of saturation at low output voltages values must be related to a phenomenon similar to those described in previous sections. 
Finally, if the short circuit current of these amplifiers were measured, a decrease from 30 to $25 \mathrm{~mA}$ at $5 \cdot 10^{13} \mathrm{n} / \mathrm{cm}^{2}$ would be found. Therefore, the saturation should be associated to the short circuit current of the operational amplifiers of the input stage rather than to the output one. This statement is supported by the fact that the output stage of these amplifiers is not improved to bias low loads. On the contrary, the output amplifier is specially designed to minimize load effects so the lower sensitivity of this amplifier is immediately deduced.

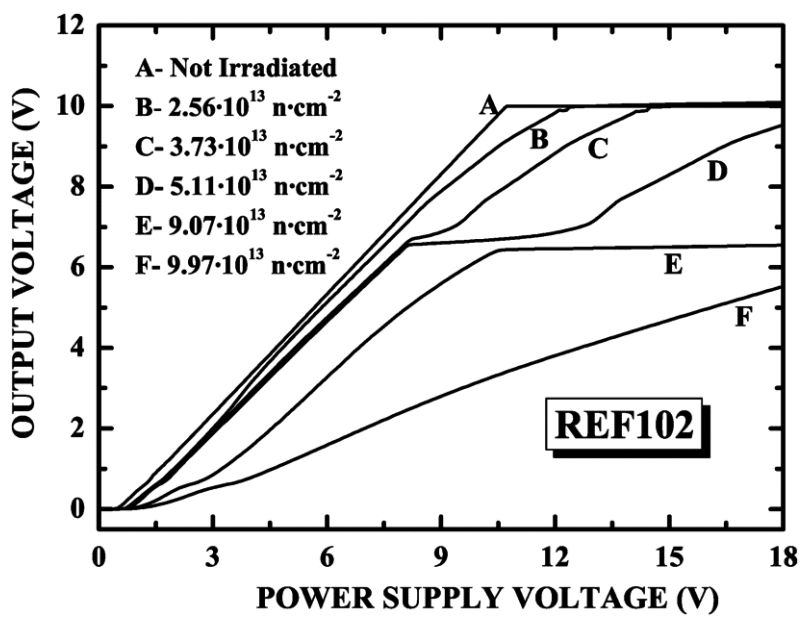

Figure 14. Transfer function between the power supply $+\mathrm{V}_{C C}$ and the output voltage in some irradiated samples of REF102 voltage reference.

3) Voltage References: These devices are designed to provide a stable output voltage. We will focus on REF102, a voltage reference from Burr-Brown with an operational amplifier and a buried Zener diode designed to provide a constant voltage of 10 V. Fig. 14 shows the output voltage as a function of the power supply on several irradiated samples. In the beginning, the function is linear if $V_{O U T}<10 \mathrm{~V}$ but, as the neutron fluence increases, the output voltage of the samples suddenly stops increasing at $7 \mathrm{~V}$ and larger supply voltages are needed to reach the nominal value.

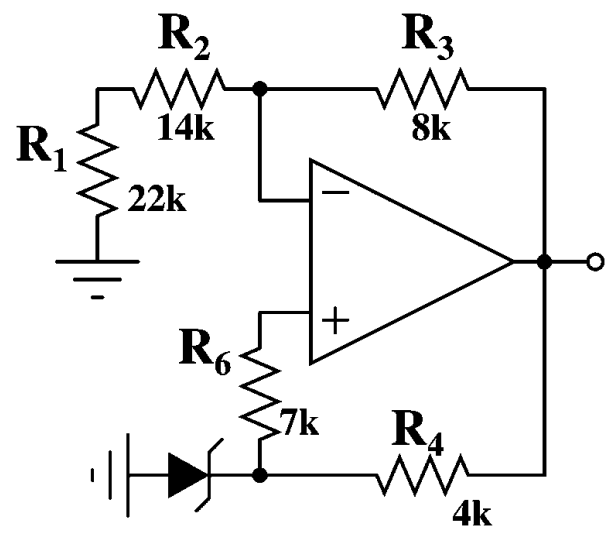

Figure 15. Internal topology of REF102 voltage reference.

This behavior can also be explained by means of the decrease of short circuit current. Fig. 15 shows the internal topology of a REF102 reference voltage. Resistors are made of metallic thin films and the Zener diode is buried in the bulk in order to minimize the noise of $\mathrm{Si}_{-} \mathrm{SiO}_{2}$ interface states [1]. It is a well known fact that these devices are extremely tolerant to radiation. Therefore, the device degradation must be related only to changes in the internal operational amplifier.

The output stage of this amplifier has a little difference with that present in previous devices: Usually, a positive voltage reference works as a supply and not as a sink so a class AB output stage is not needed. Instead of it, class A output stages are preferred (Fig. 16). However, the statement developed in Section III-A can be applied to this stage leading to a similar result: A dependence on the value of $\mathrm{h}_{F E}$ and, hence, a reduction of the positive short circuit current.

The three zones observed in the transfer function relating $+\mathrm{V}_{C C} \& \mathrm{~V}_{O U T}$ are caused by the following reasons.

1) If $+\mathrm{V}_{C C}$ is too low $(<1 \mathrm{~V})$ internal devices are not correctly biased. The amplifier cannot work and the output voltage is close to 0 .

2) If $+\mathrm{V}_{C C}$ increases some volts, the operational amplifier starts working but the output voltage is not high enough to bias the Zener diode, which must be considered as an open circuit. Consequently, the amplifier is placed in a positive 


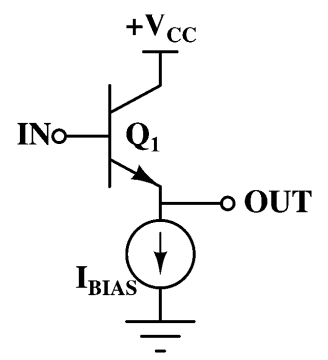

Figure 16. Class A output stage. The output current is provided by a bipolar NPN transistor $\mathrm{Q}_{1}$ which is permanently biased by a current source $\mathrm{I}_{B I A S}$.

feedback network, going the output to saturation state. In this situation, the relationship between $+\mathrm{V}_{C C}$ and $+\mathrm{V}_{O U T}$ becomes linear.

3) If $+\mathrm{V}_{C C}$ is higher than 11-12 $\mathrm{V}$, the output voltage is high enough to carry the diode into breakdown zone. Thus, the feedback becomes negative and the output voltage is stable at a value close to $10 \mathrm{~V}$.

This statement can be applied to the irradiated devices as well. However, there is a key point to be taken into account: The amplifier is biasing the feedback network. This is not important in not irradiated samples but, after the irradiation, the highest output currents of the references (and, obviously, of the internal op amp) were about $0.2-1.7 \mathrm{~mA}$ if $+\mathrm{V}_{C C}=20 \mathrm{~V}$ (Table I). This table shows the short circuit current values of samples of REF102 belonging to different batches with different power supplies values. According to the manufacturer's data sheet, the Zener diode has a breakdown voltage between 6-8 V to minimize the influence of temperature [2] and it is connected to the output of the op amp with a $4 \mathrm{k} \Omega$ resistor. Therefore, the biasing of the Zener diode needs at least $0.50-0.75 \mathrm{~mA}$, values too close to the positive short circuit current of the internal operational amplifier. The conclusion is clear: The reference cannot reach an output value of $10 \mathrm{~V}$ since the operational amplifier is not able to bias the internal feedback network.

Table I

SHORT CIRCUIT CURRENT OF IRRADIATED SAMPLES OF REF102 WITH DIFFERENT VALUES OF POWER SUPPLY. BOLD CHARACTERS ARE RELATED TO SAMPLES WHOSE OUTPUT IS $10 \mathrm{~V}$ OR MORE.

\begin{tabular}{cccc}
\hline Neutron Fluence & $V_{C C}=15 \mathrm{~V}$ & $V_{C C}=\mathbf{2 0 V}$ & $V_{C C}=\mathbf{3 0 V}$ \\
\hline Not Irradiated & 28.0 & $\mathbf{2 8 . 0}$ & $\mathbf{2 7 . 0}$ \\
\hline $1.80 \cdot 10^{13}$ & 1.48 & 1.70 & 2.50 \\
\hline $2.28 \cdot 10^{13}$ & 1.16 & 1.47 & 1.56 \\
\hline $2.76 \cdot 10^{13}$ & 0.86 & 1.01 & 1.57 \\
\hline $3.46 \cdot 10^{13}$ & 1.40 & 1.81 & 1.75 \\
\hline $3.73 \cdot 10^{13}$ & 0.86 & 1.04 & 1.28 \\
\hline $4.33 \cdot 10^{13}$ & 1.75 & 1.61 & 2.04 \\
\hline $5.11 \cdot 10^{13}$ & 0.74 & 0.87 & 1.24 \\
\hline $6.78 \cdot 10^{13}$ & 0.43 & 0.51 & 0.71 \\
\hline $7.60 \cdot 10^{13}$ & 0.59 & 0.67 & 0.90 \\
\hline $9.07 \cdot 10^{13}$ & 0.37 & 0.45 & 0.64 \\
\hline $9.95 \cdot 10^{13}$ & 0.14 & 0.17 & 0.24 \\
\hline $\mathrm{n} \cdot \mathrm{cm}^{-2}$ & $\mathrm{~mA}$ & $\mathrm{~mA}$ & $\mathrm{~mA}$ \\
\hline
\end{tabular}

Discrete op amps showed a growth of positive short circuit current if the power supplies values increased. This phenomenon also happens in the integrated amplifiers and this fact explains why the positive short circuit current increases in proportion to $+\mathrm{V}_{C C}$ (Table I). Also, this phenomenon is the reason of the increase of the minimum value of power supply to reach the theoretical output voltage of $10 \mathrm{~V}$. The positive short circuit current increases with the power supply so, when it is high enough, that current reaches the critical value of $0.75 \mathrm{~mA}$ and can bias the Zener diode.

In Table I, bold characters were used to indicate if the voltage reference reaches the theoretical value of $10 \mathrm{~V}$. If the short circuit current is lower than 1.16-1.28 mA, the reference does not reach the theoretical value. So, there is a little disagreement between the theoretical lowest current to obtain an output voltage of $10 \mathrm{~V}$ and the actual one. However, this difference can be easily explained. When the positive short circuit current is measured, the device output is connected to ground. Thus, the collector-emitter voltage of $\mathrm{Q}_{1}, \mathrm{~V}_{C E, Q 1}$ is only $V_{C C}-10$. This difference of collector-emitter voltages is very large and, in consequence, these disagreement must be also explained as a consequence of the Early effect. 
The study of the degradation of the REF102 reference is very easy because of two reasons: First of all, the internal topology of this reference is public and, second, all the internal devices are rad-tolerant except the bipolar operational amplifier. However, the results that we obtained on this reference may be extended to other similar devices, e.g., a previous work [13] describes a sudden drop from 10 to $7 \mathrm{~V}$ in the internal buried Zener reference of an D/A converter, AD565. Indeed, the topology of this device, property of Analog Devices, shows that this reference must provide $0.5 \mathrm{~mA}$ to the internal R/2R ladder network. After the safety period, we found that the short circuit current was between $0.5-1.0 \mathrm{~mA}$ so the hypothesis of the degradation of $I_{S h C C}$ is trustworthy. Also, a sudden decrease from 5 to $3 \mathrm{~V}$ was found in the reference REF02 of Burr-Brown [14], which could be also attributed to this phenomenon.

Table II

SHORT CIRCUIT CURRENT OF IRRADIATED BAND-GAP REFERENCES

\begin{tabular}{cccc}
\hline Model /Company & $V_{C C}=\mathbf{1 0 V}$ & $V_{C C}=\mathbf{2 0 V}$ & $V_{C C}=\mathbf{3 0 V}$ \\
\hline REF01 Maxim & --- & 0.55 & 0.87 \\
\hline REF02 Maxim & 3.36 & 8.90 & 19.00 \\
\hline REF02 Analog & 2.10 & 5.60 & 11.00 \\
\hline & $\mathrm{mA}$ & $\mathrm{mA}$ & $\mathrm{mA}$ \\
\hline
\end{tabular}

Finally, the internal op amp of band-gap references suffers the same degradation than that of buried Zener devices'. Indeed, some samples of AD780, REF01, and REF02 showed a large decrease of the short circuit current. Table II shows the short circuit current of some voltages references after receiving a neutron fluence of $3.28 \cdot 10^{13} \mathrm{n} / \mathrm{cm}^{2}$. Bold characters are used to underline the situations where the output voltage reached the theoretical value. In all of them, the initial value was $25 \mathrm{~mA}$ with any value of power supply. The decrease of this parameter might commit a voltage reference if it is supposed to supply a significant current.

Besides, the reduction of $I_{S h C C}$ might be related to the degradation of other references, whether band-gap or XFET type [13], [14], where large decreases in the output voltage were found. This evolution could be attributed to the decrease of short circuit current but further studies should be carried out to support this theory because, unlike buried Zener diodes, the band-gap core is sensitive to displacement damage [15].

\section{CONCLUSiOnS}

The short circuit current of irradiated bipolar operational amplifiers decreases because of the displacement damage. The reason is the reduction of the current gain of the output stage transistors, whatever the topology. If the load of the amplifier is too low, the reduction of the short circuit current might affect to the functioning of the network even though other properties of the devices had hardly changed.

Moreover, the degradation of complex devices with integrated op amps can be explained easily accepting that they suffer from a similar degradation and that the amplifiers are not able to bias the internal feedback resistor network.

\section{REFERENCES}

[1] P. R. Gray and R. G. Meyer, Analysis and Design of Analog Integrated Circuits. Wiley, 1993.

[2] D. Smith, M. Koen, and A. F. Witulski, "Evolution of high-speed operational amplifier architectures," IEEE Journal of Solid-State Circuits, vol. 29, no. 10, pp. 1166-1179, Oct 1994.

[3] E. Greeneich, Analog Integrated Circuits. Chapman and Hall, 1997.

[4] J. Marques, A. C. Fernandes, a. I. C. Gon\&ccedil, and A. J. G. Ramalho, "Test facility at the portuguese research reactor for irradiation with fast neutrons," in Proceedings of the 2004 Radiation Effects on Components and Systems Workshop, Sep. 2004.

[5] J. G. Marques, Director of Portuguese Research Reactor (private communication).

[6] "ATLAS Standard Radiation Test Methods, Appendix 2," [online] Available: http://atlas.web.cern.ch/Atlas/GROUPS/FRONTEND/radhard.htm.

[7] G. C. Messenger, "A summary review of displacement damage from high energy radiation in silicon semiconductors and semiconductor devices," IEEE Transactions on Nuclear Science, vol. 39, no. 3, pp. 468-473, Jun 1992.

[8] G. Messenger and M. Ash, "The Effects of Radiation on Electronic Systems," 1992.

[9] S. M. Sze, "Physics of Semiconductor Devices," 1981.

[10] P. Horowitz, "The Art of Electronics," 1990.

[11] L. Bonora and J. David, "An attempt to define conservative conditions for total dose evaluation of bipolar ICs," IEEE Transactions on Nuclear Science, vol. 44, no. 6, pp. 1974-1980, Dec 1997.

[12] A. J. Peyton and V. Walsh, "Analog Electronics With Op Amps. A Source Book of Practical Circuits," 1993.

[13] J. A. Agapito et al., "Radiation test on commercial instrumentation amplifiers, analog switches and DACs," pp. 113 -118, 2001.

[14] - "Instrumentation amplifiers and voltage controlled current sources for LHC cryogenic system," pp. 275-280, 2000.

[15] B. Rax, C. Lee, and A. Johnston, "Degradation of precision reference devices in space environments," IEEE Transactions on Nuclear Science, vol. 44, no. 6, pp. 1939-1944, Dec 1997. 